\title{
A GM-CSF and CD40L bystander vaccine is effective in a murine breast cancer model
}

\author{
This article was published in the following Dove Press journal: \\ Breast Cancer: Targets and Therapy \\ 15 December 2015 \\ Number of times this article has been viewed
}

\author{
Hatem Soliman' \\ Melanie Mediavilla-Varela ${ }^{2}$ \\ Scott J Antonia ${ }^{3}$ \\ 'Department of Women's Oncology \\ and Experimental Therapeutics, \\ ${ }^{2}$ Department of Immunology, \\ ${ }^{3}$ Department of Thoracic Oncology, \\ Moffitt Cancer Center, Tampa, FL, USA
}

Background: There is increasing interest in using cancer vaccines to treat breast cancer patients in the adjuvant setting to prevent recurrence in high risk situations or in combination with other immunomodulators in the advanced setting. Current peptide vaccines are limited by immunologic compatibility issues, and engineered autologous cellular vaccines are difficult to produce on a large scale. Using standardized bystander cell lines modified to secrete immune stimulating adjuvant substances can greatly enhance the ability to produce immunogenic cellular vaccines using unmodified autologous cells or allogeneic medical grade tumor cell lines as targets. We investigated the efficacy of a cellular vaccine using B78H1 bystander cell lines engineered to secrete granulocyte macrophage-colony stimulating factor and CD40 ligand (BCG) in a murine model of breast cancer.

Methods: Five-week-old female BALB/c mice were injected orthotopically in the mammary fat pad with 4T1 tumor cells. Treatment consisted of irradiated 4T1 \pm BCG cells given subcutaneously every 4 days and was repeated three times per mouse when tumors became palpable. Tumors were measured two to three times per week for 25 days. The vaccine's activity was confirmed in a second experiment using Lewis lung carcinoma (LLC) cells in C57BL/6 mice to exclude a model specific effect. Interferon- $\gamma($ IFN- $\gamma$ ) and interleukin-2 (IL-2) enzyme-linked immunospots (ELISPOTS) were performed on splenic lymphocytes incubated with 4T1 lysates along with immunohistochemistry for CD3 on tumor sections.

Results: Tumor growth was significantly inhibited in the 4T1-BCG and LLC-BCG treatment groups when compared to 4T1 and LLC treatment groups. There were higher levels of IL-2 and IFN- $\gamma$ secreting T-cells on ELISPOT for BCG treated groups, and a trend for higher numbers of tumor infiltrating CD3+ lymphocytes. Some tumors in the 4T1-BCG demonstrated organized lymphoid structures within the tumor microenvironment as well.

Conclusion: The use of BCG bystander cell lines demonstrates proof of concept for anti-tumor activity and immunogenicity in an immunocompetent murine model of breast cancer. This vaccine is being evaluated in lung cancer and should be explored further in clinical trials of breast cancer patients at high risk of recurrence or in combination with other immunomodulatory agents.

Keywords: breast cancer, immunotherapy, bystander vaccine, CD40L, GM-CSF

\section{Background and significance}

It is estimated that 232,670 patients in the USA will be diagnosed with breast cancer and 40,000 will die of their disease in 2014. While progress has been made in the diagnosis and treatment of breast cancer, there is significant room for improvement in patients with high risk, chemorefractory disease. Another challenge for some breast cancer patients is the dilemma of delayed recurrence occurring several years after the initial diagnosis. In either case, this reflects a resistance to existing treatments, which 
requires novel therapeutic strategies to improve outcomes. There is an increased appreciation for the role of the host immune response in the natural history of breast cancer. ${ }^{1,2}$ An enhanced CD8+ T-cell tumoral infiltrate predicts a better prognosis and improved response to neoadjuvant chemotherapy. ${ }^{3}$ The ability to recruit an effective anti-tumor immune response in women at risk for metastatic recurrence represents a nontoxic therapeutic option for women with these difficult to treat breast cancers. Completed and ongoing adjuvant trials using peptide based vaccines against epitopes of human epithelial receptor 2 (HER-2) shows that this approach is safe, feasible, and has shown early signs of efficacy. ${ }^{4}$ Some limitations of this approach are that peptide vaccines require patients to be human leukocyte antigen (HLA)-A2 or A3+ and the single epitope may limit the extent of the immune response. Cellular vaccines, while more labor intensive, can provide a wider range of patient tumor specific epitopes and are not HLA subtype dependent. Another non-HLA dependent method of vaccination involves anti-idiopathic antibody vaccines, which also mimic the HER-2 protein to generate anti-HER-2 immune responses. ${ }^{5,6}$ Generally, breast cancer tumor cells are not strongly immunogenic since most clones undergo immune editing selection pressure during early tumorigenesis. This makes genetic engineering of the breast cancer cells or a potent adjuvant mandatory to achieve an effective immune response.

The immune adjuvant platform selected for this study uses murine $\mathrm{B} 78 \mathrm{H} 1$ bystander cells that secrete granulocytemacrophage colony stimulating factor (GM-CSF) and CD40 ligand (CD40L) also known as BCG. The B78H1 cell line is a modified B16 melanoma murine cell line, which is deficient in major histocompatibility complex class I expression, thereby dramatically lessening its recognition by the host adaptive immune response. Secretion of GM-CSF by the bystander cells promotes activation and maturation of nearby dendritic cells (DCs) as they process antigens. Using GM-CSF secreting cell lines in cellular vaccines has been described extensively following the initial seminal work by Dranoff et al. ${ }^{7,8}$ The activity of GM-CSF secreting bystander lines spurred development of modified bystander cell lines that co-express other stimulatory molecules to further enhance the response to tumor antigens. ${ }^{9}$ One such ligand, CD40L, is able to reverse cancer-related immunosuppression of activated DCs expressing CD40 receptors leading to more potent immune responses. So researchers in $\mathrm{Dr}$ Antonia's lab created the BCG cell lines for this purpose. ${ }^{10}$ Using standardized bystander cells as the source of GM-CSF and CD40L allows the use of tumor cells from different patients as the vaccine antigen source without the need for individually genetically modifying them to secrete these factors. The BALB/c-4T1 orthotopic breast cancer model was used for this study as it is immunocompetent and 4T1 specific T-cell responses could be evaluated. A second set of experiments using C57BL/6 mice subcutaneously injected with Lewis lung carcinoma (LLC) tumors were performed to exclude model specific effects on the BCG vaccine's efficacy. The primary aim of the study was to demonstrate immune activation and anti-tumor efficacy of the BCG bystander cell line in an established breast cancer model.

\section{Materials and methods Culture of cell lines}

The murine cancer cell lines 4T1 and LLC (ATCC, Rockville, MD, USA) were maintained at $37^{\circ} \mathrm{C}$ in RPMI medium supplemented with $10 \%$ fetal calf serum, $50 \mathrm{U} / \mathrm{mL}$ penicillinstreptomycin, and $2 \mathrm{mM}$ L-glutamine (Thermo Fisher Scientific, Waltham, MA, USA) in $5 \% \mathrm{CO}_{2}$.

\section{Vaccine production}

The BCG bystander cell line was established in Dr Antonia's lab using a pCDNA3.1 plasmid with murine CD40L transfected into B78H1 GM-CSF cell lines with the FUGENE transfection system. Cells were cultured in RPMI complete media at $37^{\circ} \mathrm{C}$ and $5 \% \mathrm{CO}_{2}$ with $50 \mu \mathrm{g} / \mathrm{mL}$ hygromycin and $50 \mu \mathrm{g} / \mathrm{mL} \mathrm{G}-418$ for antibiotic selection. Expression of CD40L (BD Biosciences, San Jose, CA, USA) and GM-CSF (R\&D Systems, Inc., Minneapolis, MN, USA) in cell aliquots with their supernatant was analyzed by flow cytometry analysis and cytometric bead flow assay (BD Biosciences), respectively. The cells were left in culture flasks overnight, and the next day they were collected and frozen in aliquots of $10 \times 10^{6} / \mathrm{mL}$ in $90 \%$ fetal bovine serum and $10 \%$ dimethyl sulfoxide to be thawed on vaccination days.

\section{Murine experiments}

Five-week-old female BALB/c mice were purchased from NCI and housed in the Moffitt Cancer Center vivarium and provided ad libitum access to food and water. All animal work was conducted in accordance with all institutional guidelines following approval by the University of South Florida IACUC (Approval \# R3801). Mice in the 4T1 alone and 4T1 + BCG groups were injected with $5 \times 10^{3} 4 \mathrm{~T} 1$ cells orthotopically in the fourth mammary fat pad and tumor two-dimensional size measurements were taken two to three times per week to calculate the tumor area $(\mathrm{L} \times \mathrm{W})$. When tumors reached a mean area size of $5 \mathrm{~mm}^{2}$, the mice were randomized into 
groups of five and underwent a series of three subcutaneous injections constituting either a group injected with $2 \times 10^{6}$ irradiated 4T1 cells alone, or $2 \times 10^{6} 4 \mathrm{~T} 1+2 \times 10^{6} \mathrm{BCG}$ irradiated cells every 4 days. A third group of control mice that were not injected with any 4T1 tumor cells were used to generate tumor-naive lymphocytes as a control for the enzyme-linked immunospot (ELISPOT) analyses. Mice were euthanized 25 days after first vaccination. Lymphocytes from spleens, mesenteric, and inguinal lymph nodes were harvested and dissociated using the plunger-screen method to yield a cell suspension, which was cryopreserved for later immune activation testing. ${ }^{11}$ The experiments for the vaccine were also repeated in a similar fashion using LLC cells injected subcutaneously into 5-week-old C57BL/6 mice to confirm anti-tumor effect in a second in vivo model.

\section{IL-2 and IFN- $\gamma$ ELISPOT}

Harvested lymph nodes from each mouse were subjected to ELISPOT analysis using cytokine ELISPOT kits for mouse interleukin-2 (IL-2) and interferon- $\gamma$ (IFN- $\gamma$ ) from Mabtech (Cincinnati, OH, USA). Lymph nodes cells $\left(2.5 \times 10^{5} /\right.$ well) and irradiated $4 \mathrm{~T} 1$ lysates $\left(1.25 \times 10^{5} / \mathrm{mL}\right)$ were added into pre-coated wells of an ELISPOT plate with capture antibodies for mouse IL- 2 and IFN- $\gamma$ and incubated at $37^{\circ} \mathrm{C}$. After 48 -hour incubation, the plates were washed, and biotinylated detection antibodies for mouse IL- 2 or IFN- $\gamma$ were added to each well. The plates were washed and streptavidinhorseradish peroxidase antibody was added to each well. Following three washes, the substrate solution TMB-H (Moss, Inc., Pasadena, MD, USA) was added to each well, washed, and after an overnight drying period the spots were counted using an automated CTL ELISPOT reader.

\section{Tumor immunohistochemistry for CD3 and CDI 9 infiltrates}

Sections of harvested tumors from each mouse were placed in $10 \%$ buffered formalin for fixation and processed in the Moffitt tissue core for embedding into paraffin blocks. Ten micron sections were cut and stained using a mouse reactive anti-CD3 (Cell Marque \#103A-78, neat) and anti-CD19 (Abbiotec \#250585, 1:300), using Omnimap CC1 standard antigen retrieval on a Ventana Immunostainer XT automated immunostainer (Ventana Inc., Tuscon, AZ, USA). Tissue staining was reviewed by the core pathologist and slides were scanned into the Aperio Image analysis system for imaging processing (Leica Biosystems, Buffalo Grove, IL, USA). Images of the tumors were processed using a positive pixel count/viable tumor algorithm, which counts the number of positive staining CD3 and CD19 pixels (red pixels) and divides it by the total number of viable tumor pixels in the region of interest (blue pixels). The algorithm excludes any necrotic or empty sections of the slide image so they do not contribute to the analyzed area in the denominator. This generates a ratio of $\mathrm{CD} 3+$ and $\mathrm{CD} 19+$ pixels per area of viable tumor, which is transformed by multiplying it by $1 \times 10^{5}$ to generate a whole-number score. The score is a numerical representation of the average density of CD3+ and CD19+ staining cells across the various tumor sections from the different treatment groups.

\section{Statistical analysis}

Average tumor sizes were plotted over time and standard error bars were generated for each of the treatment groups. The terminal mean tumor sizes were analyzed using a twosided Student's $t$-test. The ELISPOT and CD infiltrate scores for the treatment groups were analyzed with a Student's $t$-test plus a Welch's correction using Prism Graphpad v6.0 (Graphpad Software, San Diego, CA, USA).

\section{Results \\ Expression of GM-CSF and CD40L in bystander cells}

The levels of GM-CSF secretion as detected by cytometric bead assay in the bystander cells were well in excess of $2,500 \mathrm{pg} / \mathrm{mL}$ as shown in Figure 1A. Surface expression of CD40L on the bystander cells was also confirmed by flow cytometry in duplicate specimens in Figure 1B.

\section{Tumor growth experiments}

The BCG vaccinated group demonstrated slower tumor growth over the treatment period with the mean tumor size at day 25 equaling $187 \mathrm{~mm}^{2}$ compared to $227 \mathrm{~mm}^{2}$ in the control group ( $P=0.04$; Figure 2 ). The results shown are representative of duplicate experiments. This effect was seen in the C57BL/6 + LLC experiment as well with a mean tumor size of $44 \mathrm{~mm}^{2}$ in the BCG vaccinated group versus $105 \mathrm{~mm}^{2}$ in the LLC alone control group ( $P=0.008$; Figure 3$)$. The vaccine was well tolerated with no significant toxicities noted in the mice during the treatment period.

\section{Immune response to vaccine}

The ELISPOT data (Figure 4) for harvested lymphocytes incubated with 4T1 lysates demonstrated a mean IFN- $\gamma$ spot count of 75.2 (95\% confidence interval [CI]: 6.7-143.8) in the 4T1 group versus 196.1 (95\% CI: 54.6-337.7) in the BCG group $(P=0.093)$. For the IL-2 ELISPOT response data, the mean spot 


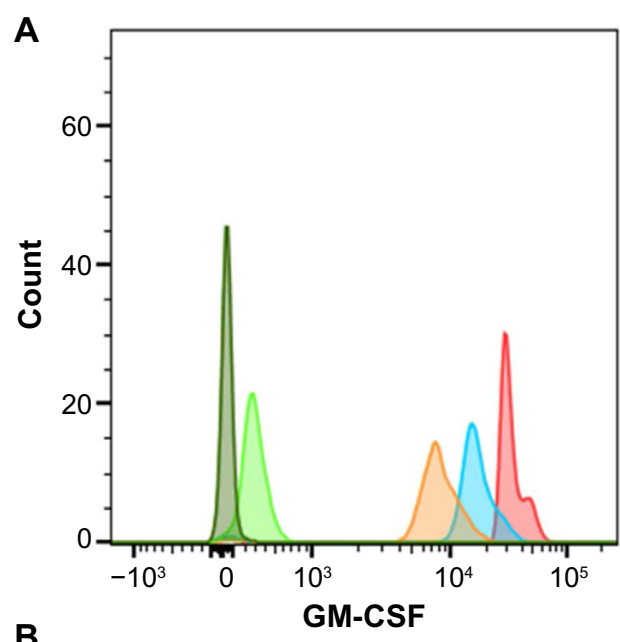

\begin{tabular}{|l|l|}
\hline & \multicolumn{1}{|c|}{ Sample name } \\
\hline$\square$ & Blank standard \\
\hline$\square$ & GM-CSF $80 \mathrm{pg} / \mathrm{mL}$ standard \\
\hline$\square$ & GM-CSF $1,000 \mathrm{pg} / \mathrm{mL}$ standard \\
\hline$\square$ & GM-CSF $2,500 \mathrm{pg} / \mathrm{mL}$ standard \\
\hline$\square$ & BCG bystander cells \\
\hline
\end{tabular}

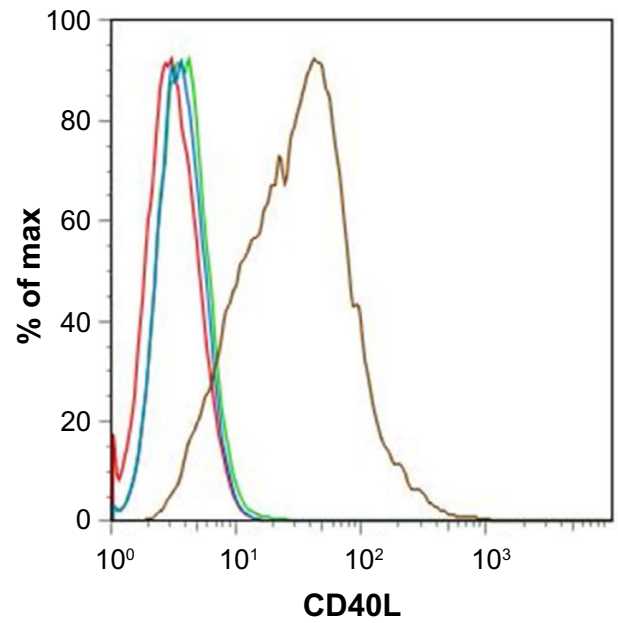

\begin{tabular}{||l|l|}
\hline & \multicolumn{1}{|c|}{ Sample name } \\
\hline & BCG stained.004 \\
\hline & BCG unstained.003 \\
\hline & Parental stained.002 \\
\hline & Parental unstained.001 \\
\hline
\end{tabular}

Figure I Characterization of bystander cell line.

Notes: (A) Flow cytometry bead analysis of GM-CSF secretion levels by the bystander cells and the standards used for quantification. The rightmost peak is the bystander cells and the peak immediately to the left of it is the $2,500 \mathrm{pg} / \mathrm{mL}$ standard. (B) Flow cytometry for surface expression of CD40L showing distinct population of positive cells with negative controls (stained and unstained parental B78HI cells plus unstained BCG cells). Data are representative of duplicate experiments for each analyte.

Abbreviations: GM-CSF, granulocyte-macrophage colony stimulating factor; CD40L, CD40 ligand.

count in the 4T1 group was 84.3 versus 287 spots in the BCG group (95\% CI: 191.2-382.8; $P=0.0013$ ). The mean number of spots from the lymphocytes in the no- tumor group treated with 4T1 lysates was 4.5 (background level). In the LLC experiment, the mean IFN- $\gamma$ spot count of LLC lysate incubated lymphocytes was 155.4 (95\% CI: 122.3-188.4) in the BCG vaccinated group versus 28.07 (95\% CI: $11.87-44.26)$ in the tumor lysate control group $(P=<0.0001)$. The IL-2 ELISPOT experiment was not repeated for the LLC mice.

\section{Tumor immune infiltrate}

The mean CD3+ count in the control group was $30.2(95 \%$ CI: 20.7-39.8) versus 62.3 (95\% CI: 31.52-92.9) in the vaccine group ( 2 sided $P=0.044$; Figure 5 ). The positive pixel/ viable tumor algorithm was able to properly identify regions of interest (shown in the blue squares), exclude necrotic zones of tumor, and identify CD3+ stained cells (red pixels on the blue background). Many of the CD3+ cells were diffusely infiltrating the tumor, but evidence of concentrated lymphoid aggregates were observed occasionally within different tumors in the BCG vaccine group. The mean CD19 (a marker of B-cells) positive pixel count in the 4T1was 4.83 (95\% CI: 2.32-7.35) versus 6.81 (95\% CI: 4.5-9.13) in the vaccine group (two-sided $P=0.182$ ).

\section{Discussions}

The main goal of this study was to generate preclinical data supporting the use of previously established bystander cell vaccines expressing GM-CSF and CD40L in an immunocompetent breast cancer model. In this regard, the data presented does demonstrate that the vaccine is able to generate tumor cell-specific Th1 responses as measured by IL- 2 and IFN- $\gamma$ 


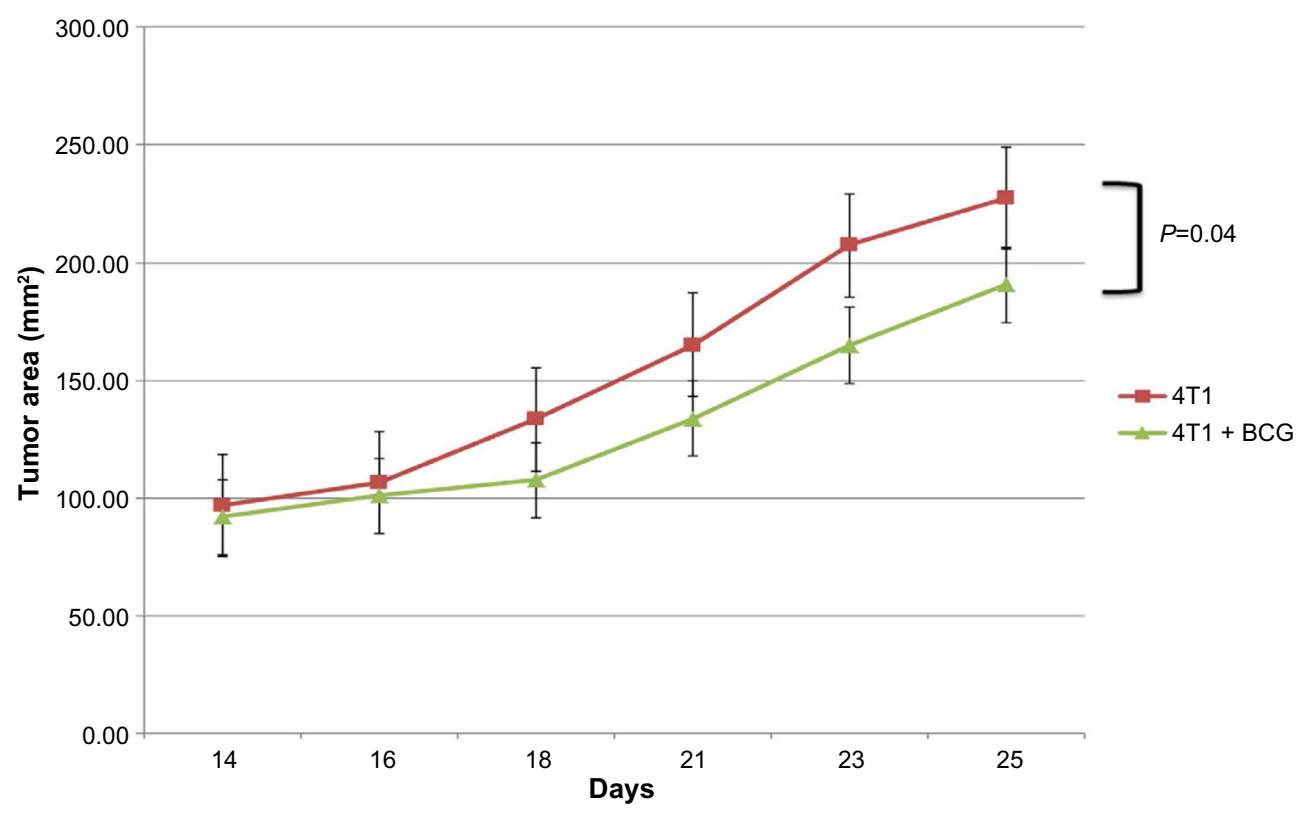

Figure 2 Tumor growth curves for 4TI alone and 4TI + BCG vaccinated groups. These results are representative of duplicate experiments. The bars display standard error.

ELISPOT tumor lysate-stimulated assays. The BCG vaccine was able to control established breast tumor growth better than using irradiated 4T1 tumor cells alone as a vaccine. This effect was observed in a second murine model using a completely different mouse strain and cell type demonstrating it is not a BALB/c - 4T1 model-specific effect. The 4T1 cell line is a very aggressive triple-negative murine ductal cancer cell line, which grows and spontaneously metastasizes quickly. This limits the number of cells (usually 5,000-20,000) that can be injected orthotopically to allow development of an adaptive immune response prior to painful tumor ulceration or excessive tumor burden mandating euthanasia. Even with relatively small numbers of cells injected when compared to other cell lines, these mice usually need to be euthanized approximately 4 weeks, which limits the length of follow-up in this mouse model. For a vaccine to show any effect on established, palpable 4T1 tumors is notable. Other available transplantable immunocompetent mouse models such as E0771 are difficult to test cellular vaccines on because they are very poorly immunogenic and are derived from a rare medullary breast cancer subtype. ${ }^{12}$ The results of this study should be confirmed in more complex experiments using

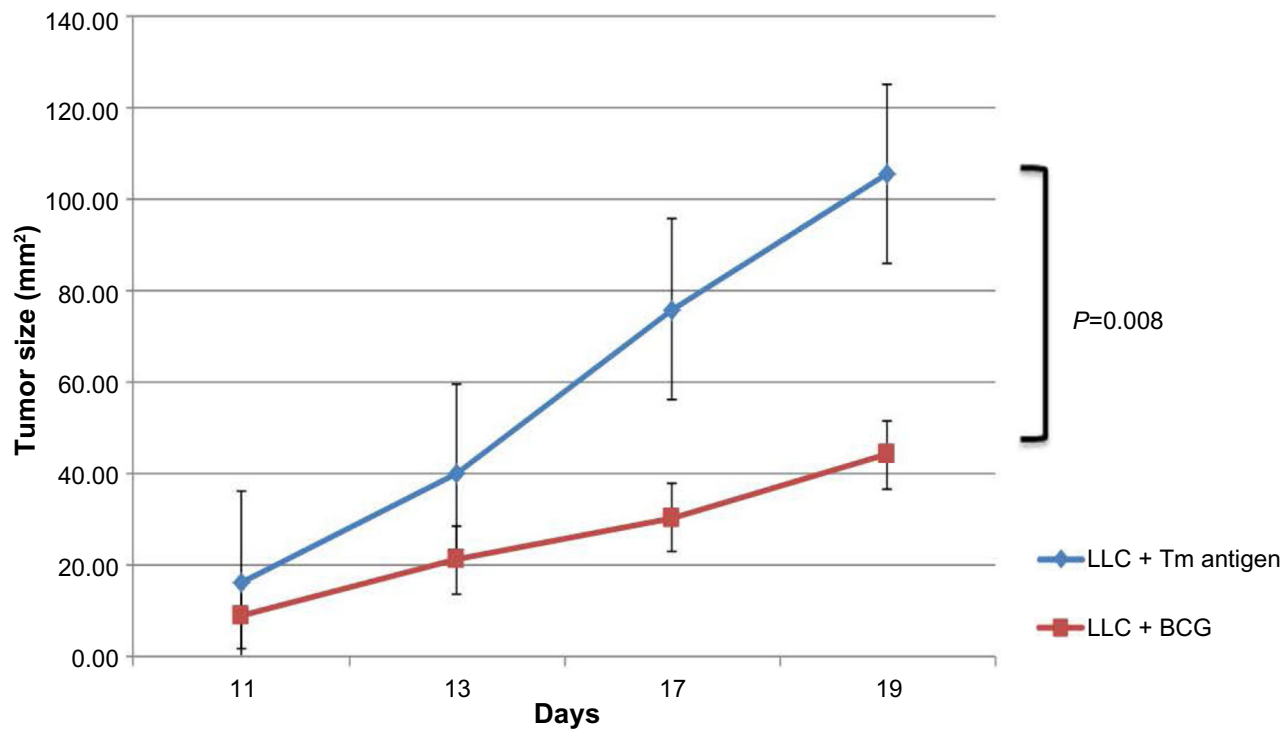

Figure 3 Tumor growth curves for LLC alone and LLC + BCG vaccinated groups. These results are representative of duplicate experiments. The bars display standard error. Abbreviation: LLC, Lewis lung carcinoma; Tm, tumor. 
A
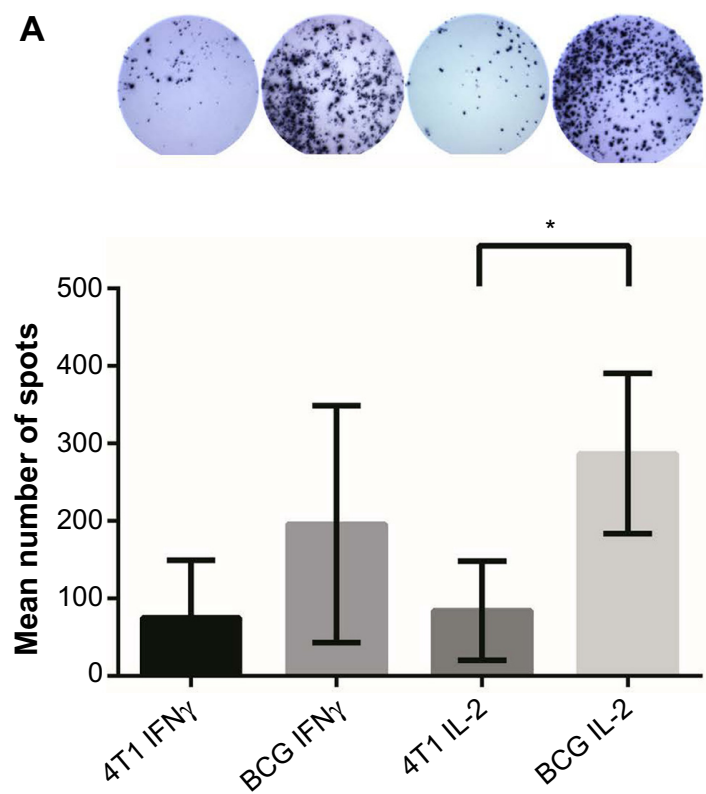

B

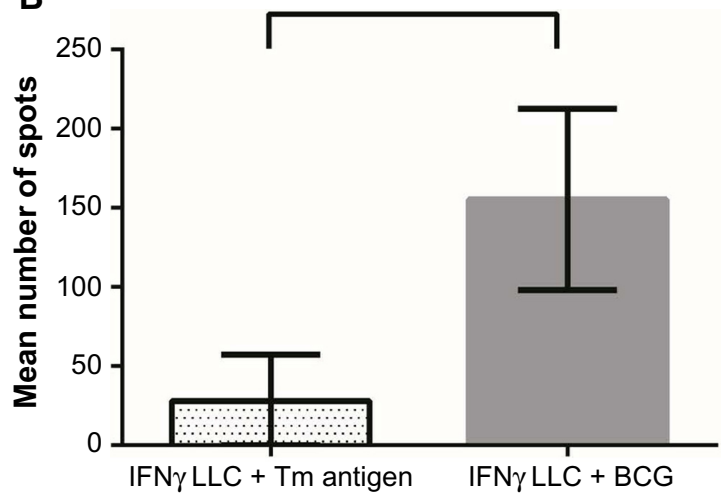

Figure 4 Immune response analysis.

Notes: (A) Average ELISPOT counts for interferon- $\gamma$ (IFN- $\gamma$ ) and interleukin-2 (IL2) between the $4 \mathrm{TI}$ alone (4TI) and 4TI + BCG (BCG) harvested lymphocytes cocultured with 4TI lysates (top). The ELISPOT images above each bar are representative of the spot counts for each of the four groups. (B) The IFN- $\gamma$ average ELISPOT counts are shown in the LLC tumor antigen alone versus the BCG vaccinated LLC mice. All experiments were done in triplicate. Spot counts in the treatment groups were adjusted for background using the no-tumor mouse lymphocytes. Error bars are the SD's for the groups. *P-value statistically significant.

Abbreviations: ELISPOT, enzyme-linked immunospot; LLC, Lewis lung carcinoma; $\mathrm{SD}$, standard deviation.

genetically engineered ductal breast cancer mouse models with derived matching cell lines that can be used as an antigen source for the vaccine along with in vivo imaging techniques. Finally, there was a trend in the tumor sections analyzed that trafficking of intratumoral CD3+ lymphocytes was enhanced in the $4 \mathrm{~T} 1+\mathrm{BCG}$ treated group over the irradiated $4 \mathrm{~T} 1$ cellalone vaccinated group. Staining mouse paraffin embedded tissues for $\mathrm{CD} 8+$ is problematic as most available antibodies demonstrate poor staining specificity. However, since CD3+ $8+$ (and not $\mathrm{CD} 4+$ ) cells are the primary mediators of antitumor immune activity, this stain can serve as a reasonable surrogate measure of cytotoxic T-cell trafficking to the tumor site. There was no evidence that the vaccine led to a greater intratumoral influx of mature CD19+ B-cells, so the observed difference in $\mathrm{CD} 3$ counts are due to increases in T-cells. Thus, this data would support the ability of the GM-CSF/CD40L bystander vaccine to generate a meaningful anti-tumor response against established breast tumors. With regards to the relative contribution of CD40L over the use of GMCSF secreting bystander cells alone, a previously published study by the Antonia's lab already addressed this question. It appears that while GM-CSF alone in mixed tumor-lymphocyte assays does increase the level of T-cell activation over control, the addition of CD40L significantly boosts this effect. ${ }^{10}$ Furthermore, published work also demonstrated the B78H1 wild-type cell line alone is totally ineffective in preventing tumor growth in BALB/c mice due to its inability to stimulate an adoptive immune response. ${ }^{9}$ Also, the existing stocks of human K562 cell lines used in human clinical trials express both GM-CSF and CD40L, so it is more relevant to show the immunologic activity of the B78H1 cell line that expresses both proteins as well. This publication also demonstrated the anti-tumor effect is mediated through the enhanced generation of tumor specific CD8+ T-cell responses. Also, previously published data by Chiodoni et al demonstrated in a BALB/c and $\mathrm{C}-26$ colon cancer model that the mechanism of action for the anti-tumor effect of GM-CSF + CD40L vaccination is enhanced antigen presentation by DCs leading to greater generation of tumor-responsive CD8+ T-cells. ${ }^{13}$ The enhanced lymphocyte trafficking within the tumors and increased Th1 cytokines seen in this study further supports this as the primary mechanism of action and there is no other plausible biologic rationale for the BCG vaccine's anti-tumor activity. Based on this study and others, the data support further studies exploring the clinical activity of K562-GMCSF-CD40L bystander vaccines in breast cancer patients with appropriate immune correlates.

\section{Translational considerations}

There are multiple considerations to take into account when considering how to use these cellular vaccines in breast cancer patients. One issue has been whether autologous tumor-derived cells or medical grade allogeneic tumor cell lines should be used as a source of target antigens. While the allogeneic cell lines can be used off the shelf quickly, there are concerns regarding whether the relevant tumor antigens present on a patient's tumor would be represented. The use of autologous tumor cells in cellular vaccines has been hampered by the difficulty in isolating sufficient primary tumor cells and added expense in generating these vaccines. This was highlighted by a recent Phase I study presented at the 2014 San Antonio Breast Conference using 


\section{A}
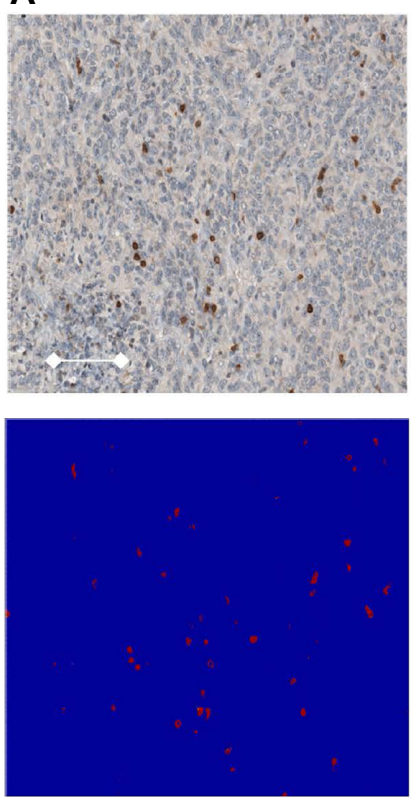

B
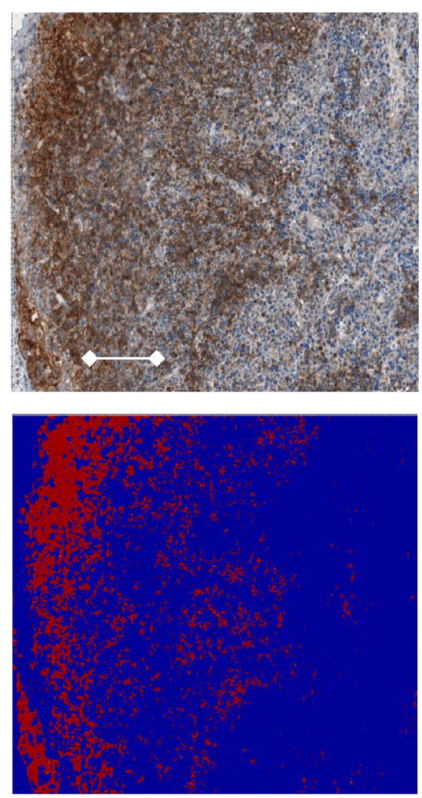

C
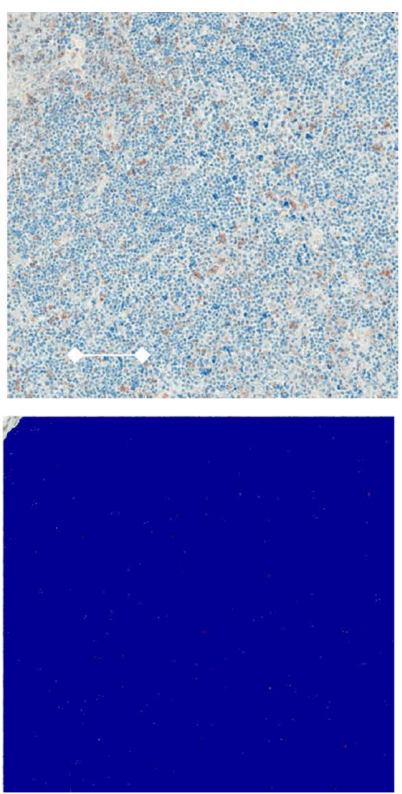

D

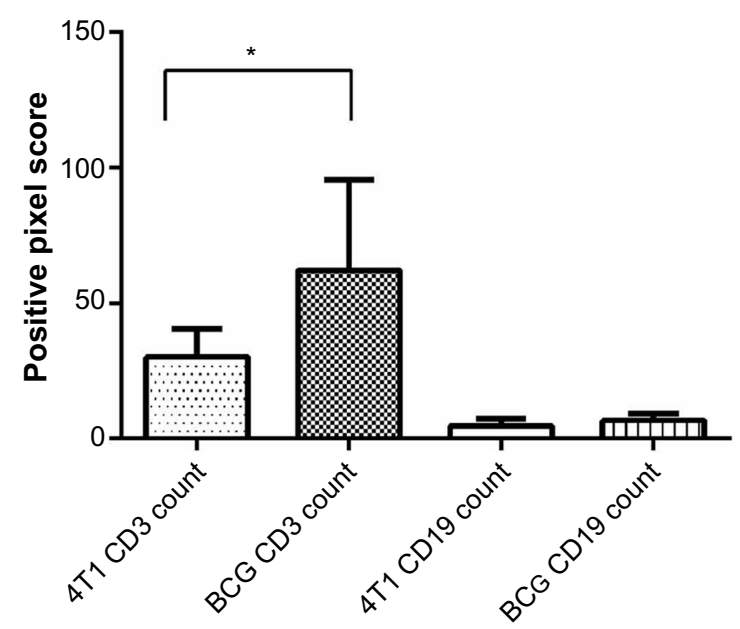

Figure 5 Immunohistochemical analysis of tumor infiltrating lymphocytes using an automated image analysis algorithm.

Notes: (A) Representative slide image showing CD3 staining in 4TI group with the red (CD3)/blue (viable tumor) image beneath demonstrating the positive pixel algorithm image annotation used for scoring. (B) Representative slide image and positive pixel algorithm image for the BCG group demonstrating significantly more CD3 positivity in the analyzed sections. The white bar on each slide image is $100 \mu \mathrm{M}$ to show scale. (C) The same CD3+ region shown in B stained for CDI9 shows very low level of B-cell infiltrates with a similar low detection in the algorithm image (D). Average CD3+ and CDI9+ pixel count scores for the 4TI and BCG tumor sections evaluated using the positive pixel count algorithm. Error bars are SD of the groups. $* P=<0.05$.

Abbreviation: SD, standard deviation.

a GM-CSF secreting autologous cellular vaccine. Out of 18 patients, only seven were able to get sufficient cells for the planned vaccinations postoperatively. One recent development, which may increase the frequency of successfully produced allogeneic cellular vaccines is the use of ROCK inhibitors and feeder cell lines to generate primary tumor cell lines pioneered by Dr Schlegel's group. ${ }^{14}$ This technique allows for the efficient and frequent generation of primary cell tumor cell lines from relatively little tumor tissue by reprogramming the cells to behave in a more stem cell-like state. This allows the cells to replicate and expand more readily in standard culture conditions. It is conceivable that this technique could be used to enhance the ability to generate sufficient amounts of autologous tumor cells that could be irradiated and combined with K562 bystander cells. Additional work would need to be done to compare the antigen expression patterns in these reprogrammed cells compared to the native tumor cells obtained from tumor specimens. The initial Phase I trial using autologous irradiated tumor cells with K562-GM-CSF-CD40L bystander cells demonstrated the vaccine was safe, immunogenic, and resulted in stabilization of disease in 13 out of 21 evaluable patients. No objective responses were noted though in this largely pretreated patient population with a median time to 
progression and overall survival of 4.5 and 24.4 months, respectively. ${ }^{15}$ Three Phase II trials have been conducted in mantle cell lymphoma, non-small cell lung cancer, and melanoma. ${ }^{16,17}$ In all these studies immunologic activity was observed, no significant toxicities were encountered, and some patients experienced stabilization or delayed progression of their disease. One melanoma patient experienced a partial response after receiving six vaccines, and another developed vitiligo secondary to the immune response from the vaccine. From this cumulative experience, it appears that while this vaccine is potent, its activity as a monotherapy is insufficient in patients with established burdens of measurable metastatic disease. In these cases, it may be beneficial to combine the vaccine with checkpoint inhibitors to further enhance the potency of anti-tumor T-cells. The recent reported activity of programmed death ligand 1 (PD-L1) blocking antibodies in triple negative breast cancer may be an ideal combination strategy. If this vaccine is used as monotherapy, it would probably be better to use it in a high risk, minimal residual disease state. One clinical scenario, which would be interesting to study is in high risk breast cancer patients (such as triple-negative ductal carcinomas) with significant residual disease present following standard neoadjuvant chemotherapy.

There is a significant unmet need in treatment refractory breast cancer patients, and the number of patients at risk for early death from metastatic recurrence is a major public health issue. The progress that has been made in cancer immunotherapy has been impressive, but additional work is needed to further enhance the number of patients who can benefit from this approach. This is especially the case in breast cancer, which is not as immunoresponsive as other tumor types, such as melanoma. However, recent data from the use of anti-PD-1/PD-L1 antibodies in triple-negative breast cancer is showing some durable clinical responses in a small proportion of patients. Safe, effective immunotherapy combinations will be required to generate substantial clinical responses for these patients. Integrating potent cellular tumor vaccines into our armamentarium will likely play an important role in improving clinical benefits of breast cancer patients undergoing immunotherapy and should be expeditiously explored in clinical trials.

\section{Acknowledgments}

This work was supported in part by the flow cytometery and tissue core facilities at the H Lee Moffitt Cancer Center and Research Institute, an NCI designated Comprehensive Cancer Center (P30-CA076292). This work was funded by the generous support of the Shula Foundation.

\section{Disclosure}

The authors report no conflicts of interest in this work.

\section{References}

1. Salgado R, Denkert C, Demaria S, et al. The evaluation of tumor-infiltrating lymphocytes (TILs) in breast cancer: recommendations by an International TILs Working Group 2014. Ann Oncol. 2014;26:259-271.

2. Ibrahim EM,Al-Foheidi ME, Al-Mansour MM, Kazkaz GA. The prognostic value of tumor-infiltrating lymphocytes in triple-negative breast cancer: a meta-analysis. Breast Cancer Res Treat. 2014;148(3):467-476.

3. Denkert C, von Minckwitz G, Brase JC, et al. Tumor-infiltrating lymphocytes and response to neoadjuvant chemotherapy with or without carboplatin in human epidermal growth factor receptor 2-positive and triple-negative primary breast cancers. J Clin Oncol. 2015;33:983-991.

4. Mittendorf EA, Clifton GT, Holmes JP, et al. Final report of the phase I/II clinical trial of the E75 (nelipepimut-S) vaccine with booster inoculations to prevent disease recurrence in high-risk breast cancer patients. Ann Oncol. 2014;25(9):1735-1742.

5. Ladjemi MZ, Chardes T, Corgnac $S$, et al. Vaccination with human antitrastuzumab anti-idiotype scFv reverses HER2 immunological tolerance and induces tumor immunity in MMTV.f.huHER2(Fo5) mice. Breast Cancer Res. 2011;13(1):R17.

6. Pal S, Saha A, Mohanty K, et al. Generation of Her-2/neu vaccine utilizing idiotypic network cascade. Cancer Biol Ther. 2007;6(12): 1916-1925.

7. Dranoff G, Jaffee E, Lazenby A, et al. Vaccination with irradiated tumor cells engineered to secrete murine granulocyte-macrophage colonystimulating factor stimulates potent, specific, and long-lasting antitumor immunity. Proc Natl Acad Sci U S A. 1993;90(8):3539-3543.

8. Eager R, Nemunaitis J. GM-CSF gene-transduced tumor vaccines. Mol Ther. 2005;12(1):18-27.

9. Borrello I, Sotomayor EM, Cooke S, Levitsky HI. A universal granulocyte-macrophage colony-stimulating factor-producing bystander cell line for use in the formulation of autologous tumor cell-based vaccines. Hum Gene Ther. 1999;10(12):1983-1991.

10. Dessureault S, Alsarraj M, McCarthy S, et al. A GM-CSF/CD40L producing cell augments anti-tumor T cell responses. J Surg Res. 2005;125(2):173-181.

11. Klein AB, Witonsky SG, Ahmed SA, et al. Impact of different cell isolation techniques on lymphocyte viability and function. $J$ Immunoassay Immunochem. 2006;27(1):61-76.

12. Ewens A, Mihich E, Ehrke MJ. Distant metastasis from subcutaneously grown E0771 medullary breast adenocarcinoma. Anticancer Res. 2005;25(6B):3905-3915.

13. Chiodoni C, Paglia P, Stoppacciaro A, Rodolfo M, Parenza M, Colombo MP. Dendritic cells infiltrating tumors cotransduced with granulocyte/ macrophage colony-stimulating factor (GM-CSF) and CD40 ligand genes take up and present endogenous tumor-associated antigens, and prime naive mice for a cytotoxic T lymphocyte response. J Exp Med. 1999;190(1):125-133.

14. Li, X, Ory V, Chapman S, et al. ROCK inhibitor and feeder cells induce the conditional reprogramming of epithelial cells. Am J Pathol. 2012;180(2):599-607.

15. Dessureault $\mathrm{S}$, Noyes D, Lee D, et al. A phase-I trial using a universal GM-CSF-producing and CD40L-expressing bystander cell line (GM.CD40L) in the formulation of autologous tumor cell-based vaccines for cancer patients with stage IV disease. Ann Surg Oncol. 2007;14(2):869-884.

16. Gray JE, Chiappori A, Williams CC, et al. A phase I/II randomized trial using GM-CSF-producing and CD40L-expressing bystander cell line (GM.CD40L) vaccine in combination with CCL21 in stage IV lung adenocarcinoma: preliminary results. J Clin Oncol. 2013;31(15): (abstr 3091).

17. Creelan BC, Antonia S, Noyes D, et al, Phase II trial of a GM-CSFproducing and CD40L-expressing bystander cell line combined with an allogeneic tumor cell-based vaccine for refractory lung adenocarcinoma. J Immunother. 2013;36(8):442-450. 


\section{Publish your work in this journal}

Breast Cancer: Targets and Therapy is an international, peer- View the full aims and scopes of this journal here. The manuscript reviewed open access journal focusing on breast cancer research, management system is completely online and includes a very quick identification of therapeutic targets and the optimal use of preven- and fair peer-review system, which is all easy to use. Visit http:// tative and integrated treatment interventions to achieve improved www.dovepress.com/testimonials.php to read real quotes from outcomes, enhanced survival and quality of life for the cancer patient. published authors.

Submit your manuscript here: http://www.dovepress.com/breast-cancer---targets-and-therapy-journal 